\title{
EFECTO DE LOS TIEMPOS DE COSECHA Y ALMACENAMIENTO EN SOLUCIÓN HIPERTÓNICA SOBRE EL COEFICIENTE DE EXPANSIÓN VOLUMÉTRICA, VOLUMEN, ESFERICIDAD Y ÁREA SUPERFICIAL DE LOS GRANOS DE MAÍZZ (Zea mai:)
}

\author{
EFFECT OF THE TIMES OF CROP AND STORAGE IN HYPERTONIC \\ SOLUTION ON THE COEFFICIENT OF VOLUMETRIC EXPANSION, VOLUME, \\ SPHERICITY AND SUPERFICIAL AREA OF THE GRAINS OF MAIZE (Zea mays)
}

\author{
${ }^{1}$ Thomas Ancco Vizcarra; ' David Juan Ramos Huallpartupa; ${ }^{2}$ Werlín Cárdenas Cañari; \\ ${ }^{3}$ Gina Genoveva Toro Rodríguez; ${ }^{3}$ Denis Hernán Gutiérrez Martinez
}

\begin{abstract}
RESUMEN
El estudio fue ejecutado en la ciudad de Andahuaylas (2926 m.s.n.m.). El objetivo fue determinar el efecto del tiempo de cosecha sobre el indice de expansión volumétrica, volumen, esfericidad y área superficial de granos de maiz de la variedad Blanco Gígante sometido a tratamiento térmico y almacenado en solución hipertónica hasta sesenta días. Se determinó que los granos cosechados a 22 días presentaron mayor relevancia en el índice de expansión volumétrica, esfericidad y área superficial, confirmando que hubo influencia en los cambios de volumen de los granos de maíz cosechados a los 28 días; asimismo se determinó que a los 45 días de almacenamiento en solución hipertónica dichos granos mostraron mayores valores en índice de expansión volumétrica, volumen, esfericidad y área superficial. Se concluyó que los tiempos de cosecha y tiempos de almacenamiento en solución hipertónica influyen en los cambios de las propiedades físicas de los granos de maíz
\end{abstract}

Palabras clave: índice de expansión volumétrica, esfericidad, área superficial, solución hipertónica.

\section{ABSTRACT}

The study was developed in Andahuaylas City, 2926 meters above sea level; the aim was determine the effect of harvest time on the rate of volumetric expansion, volume, sphericity and superficial area of grains of maize Blanco Gigante (white giant)variety subjected to heat treatment and stored in hypertonic solution up to sixty days; it was determined that the grains harvested to 22 days presented major relevancy in the volumetric index of expansion, sphericity and superficial area, the grains of maize harvested to 28 days influenced on the changes of volume; likewise, the grains showed major values in index of volumetric expansion, volume, sphericity and superficial area at 45 days of storage in hypertonic solution. It is concluded that the times of harvest and times of storage in hypertonic solution affect and influence the changes of the physical properties of grains of maize.

Key words: index of volumetric expansion, sphericity, surface area, hypertonic solution.

\section{INTRODUCCIÓN}

Actualmente, en nuestro país el maíz (Zea mays L.) es un cultivo tradicional de la sierra peruana, que constituye un componente importante de la dieta tanto de la población rural como urbana, de manera que, su importancia en la economía del poblador andino no es discutible. Por tanto, darle valor agregado representa una alternativa para mejorar la producción y productividad. El grano de maiz tierno es consumido previa cocción y al estado natural; si son cosechadas en una etapa precoz de su crecimiento (estado lechoso). La maduración del grano produce la conversión del azúcar en almidón, por lo cual el maíz dulce se conserva poco tiempo y se come fresco, o bien se comercializa enlatado o congelado, antes de que los granos se endurezcan y se vuelvan ricos en almidón; los índices de cosecha del maíz (Zea mays L.), basándose en aspectos organolépticos, y pruebas físico-químicas indican el momento exacto de la cosecha, logrando con esto la obtención del producto con buena calidad, y lograr su conservación mediante esterilización y/o apertización (Drake y Nelson, 1979). Los términos inmadurez, madurez óptima y sobre madurez se relacionan con las necesidades del mercado (Duarte, A. 1985). Carrera y Mateo (2005) mencionan que las mazorcas de maíz atraviesan tres fases de maduración: a). Madurez lechosa: El grano incrementa su peso en agua y materia seca, aumentando rápidamente su volumen, al final de esta fase los granos alcanzan su forma y peso definitivo, presenta una coloración blanca y contenido lechoso en su interior, presenta una humedad mayor al $70 \%$. b). Madurez pastosa:

Master of Sciencie en Postcosecha y Marketing, Ingeniero Agroindustrial. Facultad de Ingenieria y Instituto de Investigación y Desarrollo Tecnológico de Recursos Andinos-IIDRA de la Universidad Nacional José Maria Arguedas. Andahuaylas-Perú.

${ }^{2}$ Estudiante de Ingenieria Agroindustrial de la Universidad Nacional José María Arguedas. Andahuaylas-Perú.

${ }^{3}$ Ingeniero Agroindustrial. Facultad de Ingenieria de la Universidad Nacional José María Arguedas. Andahuaylas-Perú. 
En el interior del grano se produce un enriquecimiento de glúcidos y proteínas, la materia seca alcanza su peso máximo y el peso de agua permanece constante; el grano comienza a endurecerse, presenta una consistencia pastosa y una coloración amarillenta. Termina la movilización de las reservas hacia el grano, su contenido de agua está entre 40 y $50 \%$ sobre el peso fresco, y c). Madurez vítrea, se produce una reducción rápida del peso del grano como consecuencia de la pérdida del agua hasta alcanzar una humedad entre $12 \mathrm{y}$ $15 \%$, el grano se endurece y adopta una estructura vítrea, por lo que la madurez fisiológica se identifica por la aparición de una capa negra visible en la base del grano y para el enlatado, el grano de maíz debe tener una consistencia cremosa; el estado de maduración apropiado es entre los 15 y 19 días después de la aparición de los estigmas; al respecto, Chan (1983) indica como punto óptimo de cosecha del grano para enlatado es 20 días después de la polinización. Por otra parte, la cosecha del maíz dulce se recomienda en horas de la mañana a fin de evitar pérdida de dulzor por la conversión de los azúcares en almidón debido a altas temperaturas (Luh, 1975).

La deshidratación osmótica (DO) es una técnica que consiste en la extracción de agua de un alimento, que es sumergido en una solución hipertónica por tiempo y a temperatura específicos, esta extracción se debe a la fuerza impulsora que se crea por la alta presión osmótica donde ocurre deshidratación parcial del agua del producto, y simultáneamente se presenta en menor cantidad, la entrada de soluto desde la parte externa hasta el interior del alimento. En consecuencia el producto pierde agua, gana sólidos solubles y reduce su volumen notablemente, (Ayala et al., 2009). La DO permite aumentar la estabilidad durante el almacenamiento e incluso modificar el contenido de sólidos al final del proceso (Genina y Altamirano, 2005)

La concentración de alimentos mediante la inmersión de los mismos en una solución hipertónica se conoce como deshidratación osmótica. La ósmosis está relacionado al movimiento molecular de los componentes de una solución a través de una membrana semipermeable, hacia otra solución de menor concentración (Ibarz y Barbosa- Cánovas, 2005), y es ampliamente utilizada para la eliminación parcial del agua de los tejidos vegetales por inmersión en una solución hipertónica (osmótica). Este proceso ofrece ventajas por la mejora de las características de calidad del producto final, y la eficiencia del proceso (Sablani y Rahman, 2003). En la DO dos transferencias de masa se establecen:

a) El agua es transferida desde el producto a la solución, a menudo acompañada por sustancias naturales (azúcares, vitaminas, pigmentos y sabores).

b) La dirección opuesta, el soluto se transfiere desde la solución a las piezas de fruta (Agnelli et al., 2005)

Por otra parte, estos flujos tienen lugar en fuertes condiciones de no equilibrio, acompañado por la contracción muy importante y deformación del material osmo deshidratado (Floury et al., 2008).

Le Maguer et al., (2003), consideran que el fenómeno de transferencia de masa que ocurre en un proceso de DO es afectado por la estructura biológica y propiedades de los tejidos. En una situación ideal osmótica una membrana semi-permeable será permeable por las moléculas del disolvente pero no por las moléculas de soluto.

En los frutos o las verduras, las membranas de la pared celular poseen unidades biológicas que se pueden estirar y ampliar bajo la influencia del crecimiento y la presión de turgencia generado dentro de las células. Estas membranas celulares, que se componen principalmente de células del parénquima, no permiten que las moléculas del disolvente pasen directamente a través de ellas, sino que pasen en menor grado algunas de las moléculas de soluto. Este tipo de membrana podría ser clasificada en permeable en lugar de semi-permeable (Torregianni, 1993).

La cinética de la osmosis en alimentos puede verse afectada por: la composición química (proteínas, carbohidratos, grasa, sal, etc.), estructura física (porosidad, disposición de las células, orientación de las fibras y de la piel), y por los pre tratamientos recibidos. El comportamiento de la concentración osmótica depende de la geometría o forma de los trozos de la muestra, pues ello produce variaciones en el área superficial por unidad de volumen y en la longitud de difusión del agua y los solutos implicados en el transporte de materia. Merson y Morgan (2005) encontraron que la ganancia de soluto aumentaba si también lo hacia la relación entre el área y la forma. También hallaron que la disminución de la pérdida de agua probablemente se debe a la alta ganancia de sólidos en la superficie, que provoca la formación de una capa de soluto, y reduce la difusión. En general durante la deshidratación osmótica no es útil después de una reducción del $50 \%$ de peso, debido a la disminución en la tasa de ósmosis con respecto al tiempo. La pérdida de agua se produce principalmente durante las primeras 2 horas y la ganancia máxima sólido dentro de 30 minutos (Torreggiani et al, 1986).

\section{MATERIALES Y MÉTODOS}

Los granos de maíz variedad Blanco Gigante, fueron obtenidos del centro de producción de la Universidad Nacional José María Arguedas, cosechados a los $19,22,25,28$ y 31 días. Los mismos que fueron desgranados cuidando que la piloriza no sea dañada al momento del desgrane. Seleccionados los granos fueron acondicionados y envasados en envases de vidrio a razón de 0,250 gramos, llenándose los envases con solución hipertónica de azúcar a $16^{\circ} \mathrm{Brix}$ como liquido de gobierno; el producto fue sometido a vacío, sellado y sometido a tratamiento térmico a $121,1^{\circ} \mathrm{C}$ por 45 minutos, enfriándose inmediatamente y se almacenó, para las evaluaciones de coeficiente de expansión volumétrica, volumen, esfericidad y área superficial a los $0,15,30,45$ y 60 días respectivamente; las determinaciones del coeficiente de expansión volumétrica (Pagano y Crozza, 2001), fueron determinados por la ecuacion1, el volumen de los granos de maíz por la ecuación 2 (Mohsenin (1970) citado por Dursun (2005)), la esfericidad por la ecuación 3 (Flores, 2006), y el área superficial con la Ecuación 4 (Jain y Bal, 1997). Las determinaciones de los valores características de a, b y c de los granos de maíz fueron realizadas con pie de rey. Los resultados fueron analizados bajo un DCA con arreglo 
Ancco, T. et al., Efecto de los tiempos de cosecha y almac. en solución hipertónica sobre el coef. de expansión volumétrica, vol., esfericidad y área superf. de los granos de maíz.

factorial $5 \times 5 \times 10$, asimismo se efectuaron comparaciones múltiples de Duncan al 95\% de confianza.

$$
\begin{gathered}
Y=\begin{array}{l}
V_{g} \\
V_{g 0}
\end{array} \\
V=\frac{\pi}{6}\left(a^{*} b^{*} c\right) \\
E=\begin{array}{c}
\left(a^{*} b^{*} c\right)^{1 / 3} \\
a
\end{array} \\
S=\pi^{*} b^{*} a^{2} \\
2 a-b
\end{gathered}
$$

Dónde:

Y: Coeficiente de expansión volumétrico

\section{$\mathrm{V}$ : Volumen $\left(\mathrm{cm}^{3}\right)$}

E: Esfericidad

S: area superficial $\left(\mathrm{cm}^{2}\right)$

$\mathrm{V}_{\mathrm{R}}:$ Volumen en el tiempo de almacenamiento

$\mathrm{V}_{\mathrm{p} 0}:$ Volumen en el tiempo cero

a: $\operatorname{largo}(\mathrm{mm})$

b: ancho $(\mathrm{mm})$

c: espesor (mm)

\section{RESULTADOSYDISCUSIÓN}

Índice de expansión volumétrica de los granos de maíz en solución hipertónica

El efecto principal de tiempo de cosecha (A) y tiempo de almacenamiento (B), así como la interacción (AxB) del análisis de varianza para el índice de expansión volumétrica reflejaron diferencias estadísticas significativas

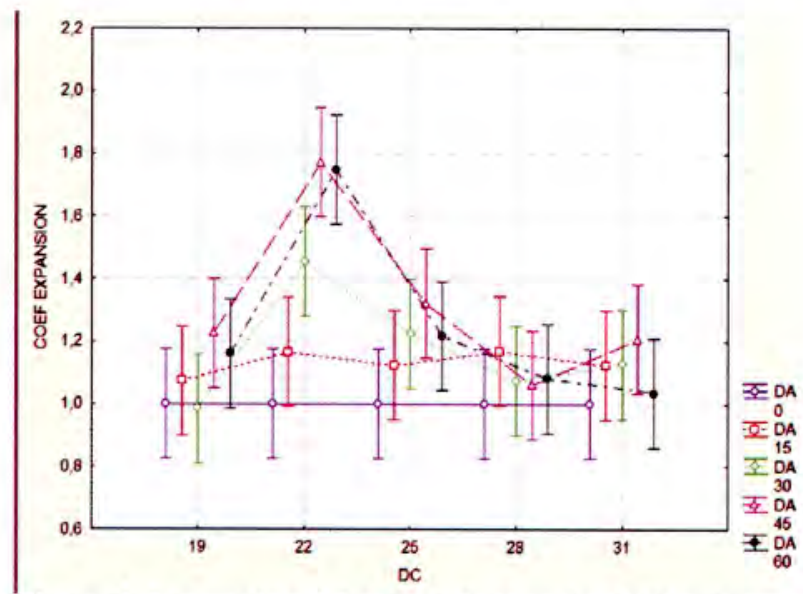

Figura 1. Interacción del tiempo de cosecha (DC) y tiempo de almacenamiento (DA) sobre el índice de expansión volumétrica de los granos de maíz en solución hipertónica al $95 \%$ de nivel de confianza, como se observa en la Figura 1, determinándose dependencia directa de la asociación del tiempo de cosecha y tiempo de almacenamiento del maíz variedad Blanco Gigante Cusco sobre el índice de expansión volumétrica, cuando estas son almacenadas en solución hipertónica. En la Tabla 1 se presenta los resultados del análisis de comparaciones múltiples de Duncan para el tiempo de cosecha, determinándose la existencia de diferencias estadísticas significativas con mayor índice de expansión volumétrica en granos de maíz cosechadas a los 22 días (1,43), a diferencia de los granos de maíz cosechados a los 25 días (1,176), 31 días (1,098), 19 días $(1,088)$ y 28 días $(1,076)$ respectivamente, no existiendo diferencias estadísticas significativas. Los efectos de la interacción $\mathrm{AxB}$ se observan en la Figura 2. Estos resultados hacen notar que a los 22 días de cosecha los granos de maíz manifiestan incremento máximo en el índice de expansión volumétrica, evidencia una variación de 0,31 respecto a los días de cosecha 25, 26 y 31 días respectivamente, como se observa en la Tabla 2 de contraste de pares de medias, muy posiblemente se atribuya también a que el tiempo de 22 días de cosecha es muy próximo al tiempo establecido por Chan (1983) y Luh (1975), y sea el más adecuado para conservar maíz en solución hipertónica a altitudes de 2926 m.s.n.m.

La Tabla 3 muestra los resultados del análisis de comparaciones múltiples de Duncan para el tiempo de almacenamiento de los granos de maíz en solución hipertónica, donde se observa que existen diferencias estadísticas significativas, determinándose mayor índice de expansión volumétrica en granos de maíz almacenados a los 45 días $(1,1317)$ y 60 días $(1,247)$; y entre los tiempos de

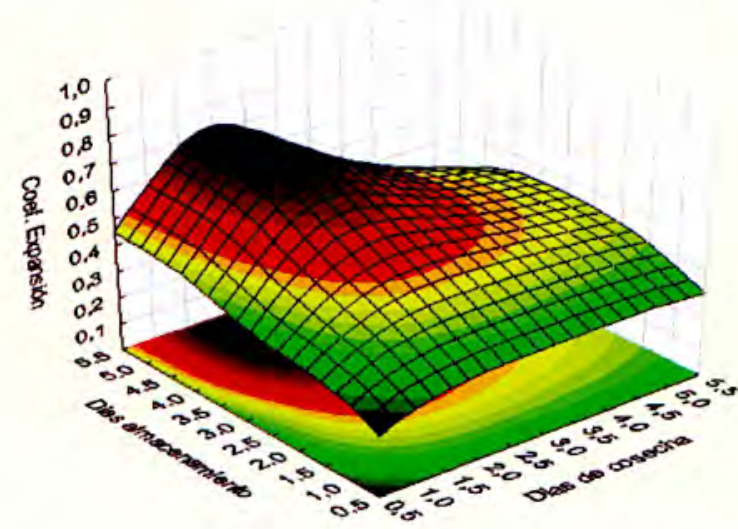

Figura 2. Efecto de tiempo de cosecha y tiempo de almacenamiento sobre el índice de expansión volumétrica en granos de maíz en solución hipertónica

Tabla 1. Pruebas de comparación de promedios del coeficiente de expansión volumétrica en solución hipertónica para el tiempo de cosecha de los granos de maíz

\begin{tabular}{ccccc}
\hline Tiempo Cosecha & Observaciones & Media LS & Sigma LS & Grupo DUNCAN \\
\hline 28 & 50 & 1,0764 & 0,0396 & $\mathrm{~b}$ \\
19 & 50 & 1,0887 & 0,0396 & $\mathrm{~b}$ \\
31 & 50 & 1,0984 & 0,0396 & $\mathrm{~b}$ \\
25 & 50 & 1,1769 & 0,0396 & $\mathrm{~b}$ \\
22 & 50 & 1,4273 & 0,0396 & $\mathrm{a}$ \\
\hline
\end{tabular}


Ancco, T. et al., Efecto de los tiempos de cosecha y almac. en solución hipertónica sobre el coef. de expansión volumétrica, vol., esfericidad y área superf. de los granos de maiz.

Tabla 2. Contraste de medias de tiempo de cosecha sobre el coeficiente de expansión volumétrica de los granos de maíz en solución hipertónica

\begin{tabular}{ccc}
\hline Contraste & Sig. & Diferencia \\
\hline $19-22$ & $*$ & $-0,3386$ \\
$19-25$ & ns & $-0,0882$ \\
$19-28$ & ns & 0,0122 \\
$19-31$ & ns & $-0,0096$ \\
$22-25$ & $*$ & 0,2503 \\
$22-28$ & $*$ & 0,3508 \\
$22-31$ & $*$ & 0,3289 \\
$25-28$ & ns & 0,1005 \\
$25-31$ & ns & 0,0785 \\
$28-31$ & ns & $-0,0219$ \\
\hline
\end{tabular}

(*) Indican diferencias significativas al 95\% de confianza

almacenamiento 30 días $(1,171), 15$ días $(1,131)$ y 0 días $(1,00)$ de almacenamiento. Los resultados del análisis de comparaciones múltiples de Duncan no mostraron diferencias estadísticas significativas al $95 \%$ de confiabilidad con margen de error estadístico del $5 \%$. Estos resultados hacen resaltar que a los cuarenta y cinco días se incrementa notoriamente el índice de expansión volumétrica en los granos de maiz, efecto atribuido al proceso de difusión osmótica de la solución hipertónica que ocasiona mayor transporte de sólidos al interior de los granos de maíz a diferencia de los 60 días donde el índice de expansión reduce por efecto de la contracción celular y eliminación de agua del interior de los granos de maíz (Torreggiani et al., 1986); asimismo la difusión ocasiona un cambio en la concentración de sólidos en el interior del grano de maíz hasta que el material llegue a un equilibrio del medio o liquido de gobierno con las semillas de maiz (Floury et al., 2008). Las diferencias determinadas en el contraste de medias entre cada grupo de estudio se puede observar en la Tabla 4, donde se resalta el mayor índice de expansión volumétrica a los 45 días de almacenamiento con una variación de $-0,188$ sobre el índice de expansión volumétrica.

\section{Volumen de los granos de maíz en solución hipertónica} Sometido a análisis estadístico los resultados del volumen de los granos de maíz en solución hipertónica, determinaron que en los efectos principales de tiempo de cosecha (A) y tiempo de almacenamiento (B), así como la interacción (AxB), hay diferencias estadísticas significativas al 95\% de nivel de confianza, efecto que se observa en la figura 3, en el que se pone en evidencia que existe una dependencia directa de la asociación del tiempo de cosecha y tiempo de almacenamiento del maíz variedad Blanco Gigante Cusco. Sobre el comportamiento del volumen de los granos de maíz almacenados en solución hipertónica, la tabla 5 presenta los resultados del análisis de comparaciones múltiples de Duncan para el tiempo de cosecha sobre el comportamiento del volumen de los granos de maíz. Se observa que existen diferencias estadísticas significativas con mayor volumen los granos de maíz cosechados a los 28 días $\left(0,718 \mathrm{~cm}^{2}\right)$ y 31 días $\left(0,713 \mathrm{~cm}^{2}\right)$. En cambio en los granos de maíz cosechados a los 22 días $\left(0,542 \mathrm{~cm}^{2}\right), 25$ días $\left(0,537 \mathrm{~cm}^{2}\right)$ y 19 dias $\left(0,437 \mathrm{~cm}^{2}\right)$ respectivamente, no se determinaron diferencias estadísticas significativas (ver figura 4). La variación entre los tiempos de cosecha de los

Tabla 3. Pruebas de comparación de promedios para el tiempo de almacenamiento de los granos de maíz sobre el Coeficiente de Expansión volumétrica en solución hipertónica

\begin{tabular}{ccccc}
\hline Tiempo Almacenamiento & Observaciones & Media LS & Sigma LS & Grupo DUNCAN \\
\hline 0 & 50 & 1,0000 & 0,0396 & $\mathrm{~d}$ \\
15 & 50 & 1,1313 & 0,0396 & $\mathrm{c}$ \\
30 & 50 & 1,1714 & 0,0396 & $\mathrm{cb}$ \\
60 & 50 & 1,2478 & 0,0396 & $\mathrm{ba}$ \\
45 & 50 & 1,3173 & 0,0396 & $\mathrm{a}$ \\
\hline
\end{tabular}

Tabla 4. Contraste de medias para el tiempo de almacenamiento sobre el coeficiente de expansión volumétrica de los granos de maíz en solución hipertónica

\begin{tabular}{ccc}
\hline Contraste & Sig. & Diferencia \\
\hline $0-15$ & $*$ & $-0,1313$ \\
$0-30$ & $*$ & $-0,1714$ \\
$0-45$ & $*$ & $-0,3172$ \\
$0-60$ & $*$ & $-0,2478$ \\
$15-30$ & ns & $-0,0401$ \\
$15-45$ & $*$ & $-0,1859$ \\
$15-60$ & $*$ & $-0,1164$ \\
$30-45$ & $*$ & $-0,1458$ \\
$30-60$ & ns & $-0,0763$ \\
$45-60$ & ns & 0,0694 \\
\hline
\end{tabular}

*indica una diferencia significativa. 
Ancco, T. et al., Efecto de los tiempos de cosecha y almac. en solución hipertónica sobre el coef. de expansión volumétrica, vol., esfericidad y área superf. de los granos de maiz

granos de maíz reflejaron diferencias estadísticas significativas. La variación promedio determinada para 28 días fue $-0,212 \mathrm{~cm}^{2}$ y para 31 días de cosecha fue $0,207 \mathrm{~cm}^{2}$. Como se observa en la Tabla 6 de contraste de pares de medias, la variación diferenciada a los 28 y 31 días de cosecha de granos de maíz posiblemente se debe a que el maíz se haya cosechado al estado lechoso o caso contrario se encontraba entre la fase pastosa y vítrea, que hace que estos cambios en el volumen se manifiesten indistintamente según el tiempo de cosecha (Carrera y Mateo, 2005), porque los granos de maíz presentan indistintamente diferente composición química, estructura física, disposición de las celulas, orientación de las fibras y diferencias en el pericarpio (Merson y Morgan, 2005).

En la Tabla 7 se presentan los resultados del análisis de comparaciones múltiples de Duncan del comportamiento del volumen de granos de maíz para el tiempo de almacenamiento en solución hipertónica, donde se observa

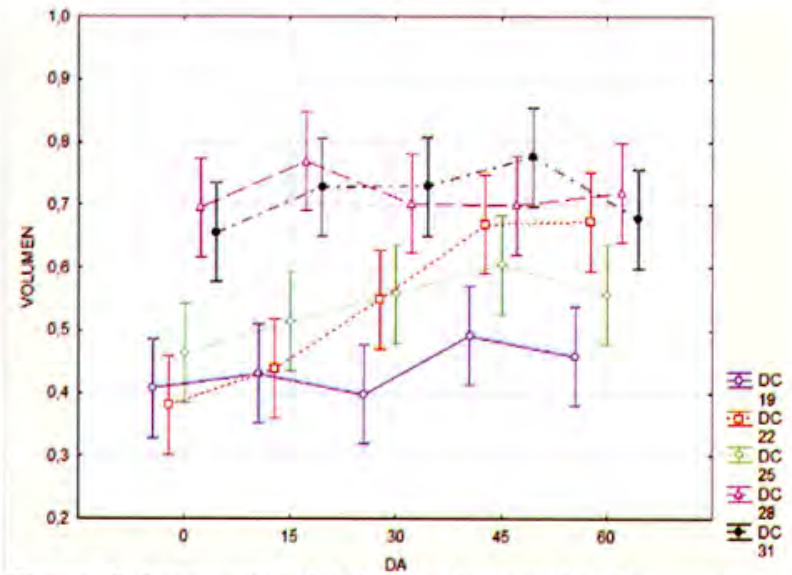

Figura 3. Interacción del tiempo de cosecha (DC) y tiempo de almacenamiento (DA) sobre el comportamiento del volumen de los granos de maíz en solución hipertónica que existen diferencias estadísticas significativas con mayor incremento de volumen los granos de maíz almacenados a los 45 días $\left(0,648 \mathrm{~cm}^{3}\right)$ y 60 días $\left(0,616 \mathrm{~cm}^{3}\right)$; entre los tiempo de almacenamiento 30 días $\left(0,587 \mathrm{~cm}^{3}\right), 15$ días $\left(0,576 \mathrm{~cm}^{3}\right)$. Los resultados de análisis de comparaciones múltiples de Duncan no mostraron diferencias estadísticas significativas al $95 \%$ de probabilidades, a diferencia de 0 días $\left(0,520 \mathrm{~cm}^{3}\right)$ con un margen de error estadístico del $5 \%$. Estos resultados hacen resaltar que a los cuarenta y cinco días incrementa notoriamente el volumen en granos de maíz en la solución hipertónica a diferencia de los 60 días donde el volumen de granos de maíz mostró 0,0312916 de variación, posiblemente debido al efecto osmótico que tiene la solución hipertónica. Lo que hace que hasta los 45 días la capacidad de osmosis está en su punto máximo y a partir de ahí se manifiesta una contracción en la estructura celular de los granos de maíz, por el cual se reduce el volumen, debido posiblemente a la fuerza impulsora de la alta presión

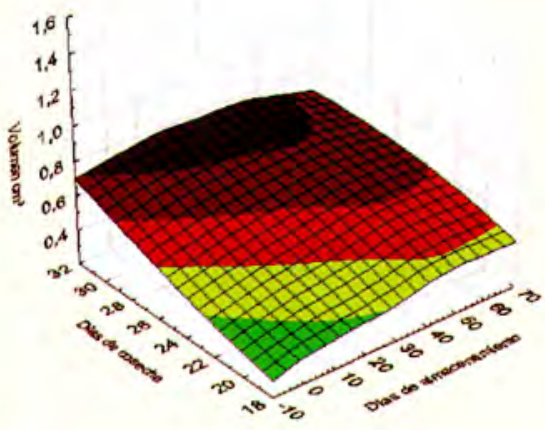

Figura 4. Efecto de tiempo de cosecha y tiempo de almacenamiento sobre el comportamiento del volumen de los granos de maíz en solución hipertónica

Tabla 5. Pruebas de comparación de promedios para el tiempo de cosecha sobre el comportamiento del volumen de los granos de maíz en solución hipertónica

\begin{tabular}{ccccc}
\hline Tiempo Cosecha & Observaciones & Media LS & Sigma LS & Grupo DUNCAN \\
\hline 19 & 50 & 0,4372 & 0,0178 & $\mathrm{c}$ \\
25 & 50 & 0,5377 & 0,0178 & $\mathrm{~b}$ \\
22 & 50 & 0,5427 & 0,0178 & $\mathrm{~b}$ \\
31 & 50 & 0,7132 & 0,0178 & $\mathrm{a}$ \\
28 & 50 & 0,7180 & 0,0178 & $\mathrm{a}$ \\
\hline
\end{tabular}

Tabla 6. Contraste de medias de tiempo de cosecha sobre el comportamiento del volumen de los granos de maíz en solución hipertónica

\begin{tabular}{ccc}
\hline Contraste & Sig. & Diferencia \\
\hline $19-22$ & $*$ & $-0,1055$ \\
$19-25$ & $*$ & $-0,1005$ \\
$19-28$ & $*$ & $-0,2808$ \\
$19-31$ & $*$ & $-0,2760$ \\
$22-25$ & ns & 0,0049 \\
$22-28$ & $*$ & $-0,1753$ \\
$22-31$ & $*$ & $-0,1705$ \\
$25-28$ & $*$ & $-0,1802$ \\
$25-31$ & $*$ & $-0,1755$ \\
$28-31$ & $\mathrm{~ns}$ & 0,0047 \\
\hline
\end{tabular}

(*) Indican diferencias significativas al $95 \%$ de confianza 
Ancco, T. et al., Efecto de los tiempos de cosecha y almac. en solución hipertónica sobre el coef. de expansión volumétrica, vol., esfericidad y área superf. de los granos de maiz.

Tabla 7. Pruebas de comparación de promedios del comportamiento del volumen de los granos de maíz en solución hipertónica durante el tiempo de almacenamiento

\begin{tabular}{ccccc}
\hline Tiempo Almacenamiento & Observaciones & Media LS & Sigma LS & Grupo DUNCAN \\
\hline 0 & 50 & 0,5207 & 0,0178 & $\mathrm{c}$ \\
15 & 50 & 0,5760 & 0,0178 & $\mathrm{~b}$ \\
30 & 50 & 0,5871 & 0,0178 & $\mathrm{~b}$ \\
60 & 50 & 0,6168 & 0,0178 & $\mathrm{ba}$ \\
45 & 50 & 0,6481 & 0,0178 & $\mathrm{a}$ \\
\hline
\end{tabular}

Tabla 8. Contraste de medias del comportamiento de volumen de los granos de maíz en solución hipertónica durante el tiempo de almacenamiento

\begin{tabular}{ccc}
\hline Contraste (Tiempo) & Sig. & Diferencia \\
\hline $0-15$ & $*$ & $-0,0552$ \\
$0-30$ & $*$ & $-0,0664$ \\
$0-45$ & $*$ & $-0,1274$ \\
$0-60$ & $*$ & $-0,0961$ \\
$15-30$ & $\mathrm{Ns}$ & $-0,0111$ \\
$15-45$ & $*$ & $-0,0721$ \\
$15-60$ & $\mathrm{Ns}$ & $-0,0408$ \\
$30-45$ & $*$ & $-0,0609$ \\
$30-60$ & $\mathrm{Ns}$ & $-0,0296$ \\
$45-60$ & $\mathrm{Ns}$ & 0,0312
\end{tabular}

* indica una diferencia significativa.

osmótica ocasionado a los 45 días. Esto es de sumo interés cuando se desea conservar adecuadamente los granos de maíz, pues este fenómeno garantiza la estabilidad del producto (Ayala et al., 2009 y Genina y Altamirano, 2005) . Las diferencias determinadas en el contraste de medias entre cada grupo de estudio se puede observar en la Tabla 8, resaltando en ella la diferencia de $0,086 \mathrm{~cm}^{2}$ a los 45 días de almacenamiento de los granos de maíz en solución hipertónica frente a los demás tiempos de almacenamiento.

\section{Esfericidad de los granos de maíz en solución hipertónica}

El efecto principal de tiempo de cosecha (A) y tiempo de almacenamiento (B) así como la interacción (AxB) del análisis de varianza para el comportamiento de la esfericidad en los granos de maíz en solución hipertónica, reportaron diferencias estadísticas significativas al 95\% de

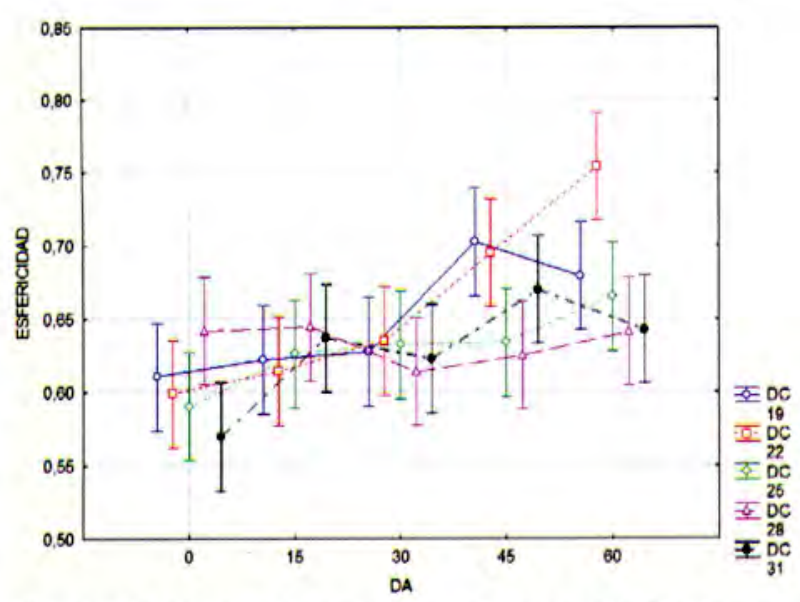

Figura 5. Interacción del tiempo de cosecha (DC) y tiempo de almacenamiento (DA) sobre el comportamiento de la esfericidad de los granos de maíz en solución hipertónica nivel de confianza, efecto que se observa en la Figura 5, evidenciando que existe una dependencia directa de la asociación del tiempo de cosecha y tiempo de almacenamiento del maíz variedad Blanco Gigante Cusco. En la tabla 9 se presentan los resultados del análisis de comparaciones múltiples de Duncan para el tiempo de cosecha sobre el comportamiento de la esfericidad de los granos de maíz, determinándose diferencias estadísticas significativas con mayor esfericidad en los granos de maíz cosechadas a los 22 días $(0,659)$ (efecto observado en la figura 6). En tanto que los granos de maíz cosechados a los 19 días $(0,648), 28$ días $(0,633), 25$ días $(0,6288), 31$ días $(0,6283)$, no presentaron diferencias estadísticas significativas. A 22 días de cosecha la esfericidad muestra notorio incremento por el efecto osmótico que hace que los granos se hinchen ampliando sus dimensiones a, b y c; esto referente en el incremento de la esfericidad con una

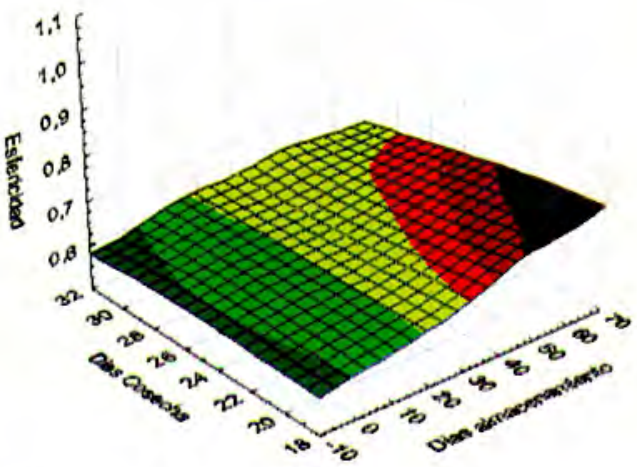

Figura 6. Efecto de tiempo de cosecha y tiempo de almacenamiento sobre el comportamiento de la esfericidad de los granos de maíz en solución hipertónica 
Ancco, T. et al., Efecto de los tiempos de cosecha y almac. en solución hipertónica sobre el coef. de expansión volumétrica, vol., esfericidad y área superf. de los granos de maíz.

Tabla 9. Pruebas de comparación de promedios para el tiempo de cosecha sobre el comportamiento de la esfericidad de los granos de maíz en solución hipertónica

\begin{tabular}{ccccc}
\hline Tiempo Cosecha & Observaciones & Media LS & Sigma LS & Grupo DUNCAN \\
\hline 31 & 50 & 0,6283 & 0,00836 & $\mathrm{~b}$ \\
25 & 50 & 0,6288 & 0,00836 & $\mathrm{~b}$ \\
28 & 50 & 0,6333 & 0,00836 & $\mathrm{~b}$ \\
19 & 50 & 0,6481 & 0,00836 & $\mathrm{ba}$ \\
22 & 50 & 0,6595 & 0,00836 & $\mathrm{a}$ \\
\hline
\end{tabular}

Tabla 10. Contraste de medias de tiempo de cosecha sobre el comportamiento de la esfericidad de los granos de maíz en solución hipertónica

\begin{tabular}{ccc}
\hline Contraste & Sig. & Diferencia \\
\hline $19-22$ & Ns & $-0,0113$ \\
$19-25$ & Ns & 0,0193 \\
$19-28$ & Ns & 0,0148 \\
$19-31$ & Ns & 0,0197 \\
$22-25$ & $*$ & 0,0306 \\
$22-28$ & $*$ & 0,0261 \\
$22-31$ & $*$ & 0,0311 \\
$25-28$ & Ns & $-0,0045$ \\
$25-31$ & Ns & 0,0004 \\
$28-31$ & Ns & 0,0049 \\
\hline
\end{tabular}

* indican diferencias significativas al $95 \%$ de confianza

Tabla 11. Pruebas de comparación de promedios del comportamiento de la esfericidad de los granos de maíz en solución hipertónica durante el tiempo de almacenamiento

\begin{tabular}{ccccc}
\hline Tiempo Almacenamiento & Observaciones & Media LS & Sigma LS & Grupo DUNCAN \\
\hline 0 & 50 & 0,6021 & 0,00836 & $\mathrm{c}$ \\
30 & 50 & 0,6261 & 0,00836 & $\mathrm{~b}$ \\
15 & 50 & 0,6286 & 0,00836 & $\mathrm{~b}$ \\
45 & 50 & 0,6651 & 0,00836 & $\mathrm{a}$ \\
60 & 50 & 0,6762 & 0,00836 & $\mathrm{a}$ \\
\hline
\end{tabular}

Tabla 12. Contraste de medias del comportamiento de la esfericidad de los granos de maíz en solución hipertónica durante el tiempo de almacenamiento

\begin{tabular}{ccc}
\hline Contraste (Tiempo) & Sig. & Diferencia \\
\hline $0-15$ & $*$ & $-0,0264$ \\
$0-30$ & $*$ & $-0,0239$ \\
$0-45$ & $*$ & $-0,0629$ \\
$0-60$ & $*$ & $-0,0741$ \\
$15-30$ & Ns & 0,0025 \\
$15-45$ & $*$ & $-0,0364$ \\
$15-60$ & $*$ & $-0,0476$ \\
$30-45$ & $*$ & $-0,0389$ \\
$30-60$ & $*$ & $-0,0501$ \\
$45-60$ & Ns & $-0,0111$ \\
\hline
\end{tabular}

* indica una diferencia significativa.

variación promedio de 0,029 a los 22 días de cosecha frente a 25,28 y 31 días de cosecha, variación que se observa en la tabla 10 de contraste de pares de medias. Estas variaciones observadas a medida que pasa el tiempo de almacenamiento, son atribuidos a la estructura biológica y propiedades de los tejidos, que se pueden estirar y ampliar bajo la presión osmótica generada por la solución hipertónica (Torregianni, 1993).

En la tabla 11 se presentan los resultados del análisis de comparaciones múltiples de Duncan del comportamiento de la esfericidad alcanzado en los granos de maíz durante el tiempo de almacenamiento en solución hipertónica, determinándose diferencias estadísticas significativas con mayor incremento de esfericidad en los granos de maíz almacenados a los 60 días $(0,676)$ y 45 días $(0,665)$; a diferencia de los tiempos de almacenamiento de 15 días $(0,628), 30$ días $(0,626)$ respectivamente, donde los resultados del análisis de comparaciones múltiples de Duncan no mostraron diferencias estadísticas significativas al 95\% de probabilidades. Las diferencias determinadas a 60 días de almacenamiento fue en promedio $-0,057$ y para 45 días -0,046 en promedio, como se observa en la tabla 12 de contraste de pares de medias. Los cambios en la esfericidad de los granos de maíz almacenados en solución hipertónica, 
muestran interés comercial, ya que la presentación de los granos se ve mejorada notoriamente respecto a su calidad (Sablani y Rahman, 2003), ofreciéndose al consumidor un producto casi uniforme, y más atractivo.

\section{Área superficial de los granos de maíz en solución hipertónica}

El efecto principal de tiempo de cosecha (A) y tiempo de almacenamiento (B) del análisis de varianza para el comportamiento del área superficial en los granos de maíz en solución hipertónica, reportó diferencias estadísticas significativas al $95 \%$ de nivel de confianza, efecto que no se determinó para la interacción (AxB), como se observa en la figura 7 , evidenciando que no existe una dependencia directa de la asociación del tiempo de cosecha y tiempo de almacenamiento del maíz variedad Blanco Gigante Cusco, esto hace notar que el área superficial de los granos de maíz

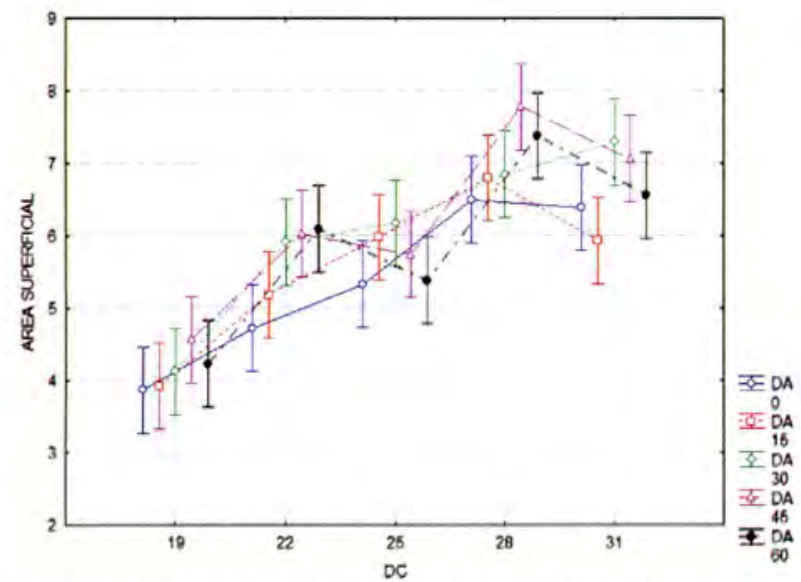

Figura 7. Interacción del tiempo de cosecha (DC) y tiempo de almacenamiento (DA) sobre el comportamiento del área superficial de los granos de maíz en solución hipertónica al ser sometidos a solución hipertónica dependerán del tiempo de cosecha y tiempo de almacenamiento (Osmosis). En la tabla 13 se presentan los resultados del análisis de comparaciones múltiples de Duncan para el tiempo de cosecha sobre el comportamiento del área superficial de los granos de maíz, determinándose diferencias estadísticas significativas con mayor área superficial alcanzada en los granos de maíz cosechadas a los 22 días $\left(0,659 \mathrm{~m}^{2}\right)$. A diferencia de los granos de maíz cosechados a los 19 días $\left(0,648 \mathrm{~m}^{2}\right), 28$ días $\left(0,633 \mathrm{~m}^{2}\right), 25$ días $\left(0,6288 \mathrm{~m}^{2}\right)$ y 31 días $\left(0,62839 \mathrm{~m}^{2}\right)$ respectivamente, los granos cosechados en los tiempos indicados no influenciaron sobre el área superficial al someterse las semillas de maíz a solución hipertónica, efecto observado en la figura 8 . El mayor incremento en el área superficial manifestado durante el almacenamiento en solución hipertónica para los granos cosechados a los 22 días en promedio fue $0,029 \mathrm{~m}^{2}$ como se observa en la tabla

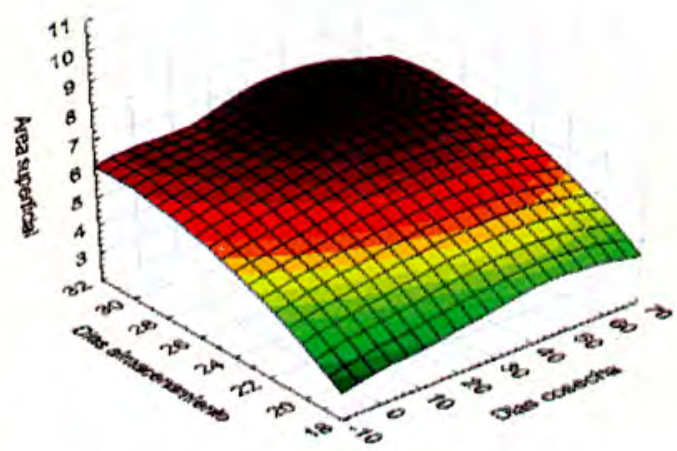

Figura 8. Efecto de tiempo de cosecha y tiempo de almacenamiento sobre el comportamiento del área superficial de los granos de maíz en solución hipertónica

Tabla 13. Pruebas de comparación de promedios para el tiempo de cosecha sobre el comportamiento del área superficial de los granos de maíz en solución hipertónica

\begin{tabular}{ccccc}
\hline Tiempo Cosecha & Observaciones & Media $L S$ & Sigma LS & Grupo DUNCAN \\
\hline 31 & 50 & 0,6283 & 0,00836 & $\mathrm{~b}$ \\
25 & 50 & 0,6288 & 0,00836 & $\mathrm{~b}$ \\
28 & 50 & 0,6333 & 0,00836 & $\mathrm{~b}$ \\
19 & 50 & 0,6481 & 0,00836 & $\mathrm{ba}$ \\
22 & 50 & 0,6595 & 0,00836 & $\mathrm{a}$ \\
\hline
\end{tabular}

Tabla 14. Contraste de medias de tiempo de cosecha sobre el comportamiento del área superficial de los granos de maíz en solución hipertónica

\begin{tabular}{ccc}
\hline Contraste & Sig. & Diferencia \\
\hline $19-22$ & Ns & $-0,0113$ \\
$19-25$ & Ns & 0,0193 \\
$19-28$ & Ns & 0,0148 \\
$19-31$ & Ns & 0,0197 \\
$22-25$ & $*$ & 0,0306 \\
$22-28$ & $*$ & 0,0261 \\
$22-31$ & $*$ & 0,0311 \\
$25-28$ & Ns & $-0,0045$ \\
$25-31$ & Ns & 0,0004 \\
$28-31$ & Ns & 0,0049 \\
\hline
\end{tabular}

* Indican diferencias significativas al $95 \%$ de confianza 
Tabla 15. Pruebas de comparación de promedios del comportamiento del área superficial de los granos de maíz en solución hipertónica durante el tiempo de almacenamiento

\begin{tabular}{ccccc}
\hline Tiempo Almacenamiento & Observaciones & Media LS & Sigma LS & Grupo DUNCAN \\
\hline 0 & 50 & 0,6021 & 0,00836 & $\mathrm{c}$ \\
30 & 50 & 0,6261 & 0,00836 & $\mathrm{~b}$ \\
15 & 50 & 0,6286 & 0,00836 & $\mathrm{~b}$ \\
45 & 50 & 0,6651 & 0,00836 & $\mathrm{a}$ \\
60 & 50 & 0,6762 & 0,00836 & $\mathrm{a}$ \\
\hline
\end{tabular}

Tabla 16. Contraste de medias del comportamiento del área superficial de los granos de maíz en solución hipertónica durante el tiempo de almacenamiento

\begin{tabular}{ccc}
\hline Contraste (Tiempo) & Sig. & Diferencia \\
\hline $0-15$ & $*$ & $-0,0264$ \\
$0-30$ & $*$ & $-0,0239$ \\
$0-45$ & $*$ & $-0,0629$ \\
$0-60$ & $*$ & $-0,0741$ \\
$15-30$ & Ns & 0,0025 \\
$15-45$ & $*$ & $-0,0364$ \\
$15-60$ & $*$ & $-0,0476$ \\
$30-45$ & $*$ & $-0,0389$ \\
$30-60$ & $*$ & $-0,0501$ \\
$45-60$ & Ns & $-0,0111$ \\
\hline
\end{tabular}

(*) Indican una diferencia significativa

14 de contraste de pares de medias. Cambios atribuidos al proceso osmótico de transferencia de masa hacia el interior, facilitado por el tiempo de cosecha de los granos donde la estructura propia de los granos está en pleno proceso de completar su formación y almacenamiento de órganos de reserva, y por lo tanto, sus membranas de la pared celular son semipermeables a los gradientes de presión osmótica (Carrera y Mateo, 2005 y Sablani y Rahman, 2003).

En la tabla 15 se presentan los resultados del análisis de comparaciones múltiples de Duncan del comportamiento del área superficial de los granos de maíz durante el tiempo de almacenamiento en solución hipertónica, determinándose diferencias estadísticas significativas con mayor incremento de área superficial en los granos de maíz almacenados a los 60 días $(0,676)$ y 45 días $(0,665)$; a diferencia de los tiempos de almacenamiento de 15 días $(0,628)$, 30 días $(0,626)$. Los resultados hacen resaltar que a los sesenta y cuarenta y cinco días incrementa notoriamente el área superficial en los granos de maíz dentro de la solución hipertónica, efecto que posiblemente se deba al efecto osmótico que incita a un agrandamiento de los granos de maíz hasta lograr un máximo dentro del tiempo estudiado. Las diferencias determinadas para 60 días de almacenamiento en promedio fue $-0,057 \mathrm{~m}^{2}$ y para 45 días $0,046 \mathrm{~cm}^{2}$ en promedio, como se observa en la tabla $16 \mathrm{de}$ contraste de pares de medias, incremento atribuido a la tasa osmótica en el tiempo que ocasiona el transporte de materia al interior de los granos de maíz, provocando variación en el área superficial por unidad de volumen (Merson y Morgan, 2005).

\section{CONCLUSIONES}

El tiempo de cosecha de los granos de maíz variedad Blanco Gigante Cusco, influye en el índice de expansión, volumen, esfericidad y área superficial del maíz almacenado en solución hipertónica.

Los tiempos de almacenamiento en solución hipertónica de los granos de maíz influyen con cambios hasta los 45 días de almacenamiento en el índice de expansión, incremento de volumen, modificaciones en la esfericidad, área superficial.

\section{REFERENCIAS BIBLIOGRÁFICAS}

Agnelli, M. E., Marani, C. M., and Mascheroni, R. H. 2005. Modelling of heat and mass transfer during (osmo)dehydrofreezing of fruits. Journal of Food Engineering, 69, 415-424.

Ayala Aponte, Alfredo Adolfo, Serna Cock, Liliana y Giraldo-Cuartas, Carlos Julián. Efecto de la agitación sobre la deshidratación osmótica de pitahaya amarilla (selenicereus megalanthus s.) Empleando soluciones de sacarosa. 2009. INCI, vol.34, no.7, p. 492-496. ISSN 0378-1844.

Carrera, M. y Mateo, J., 2005, Prontuario de agricultura, Cultivos Agricolas. E. Aedos, Mexico D.F., Mxico, pp.18-20.

Chan, P. The relation of maturity of sweet com to the quality ofthe canned product. Scientific meeting report 1982. Tainan District Agricultural Improvement Station (1983). 111-117. Field Crop Abstracts. 1984. N 5254 .

Drake, S.R. y Nelson, J.W. (1979). A comparison of three metbods of maturity determination in sweet corn. Hostscience 14(4): 546-548.

Duarte, A. (1985). Caracteristicas quimicas que afectan el rendimiento y la calidad en la molienda de algunos cultivares de maiz. Maracay, V zla. Tesis. Fac. Agronomía, UCV. $107 \mathrm{p}$.

Dursun, I.; Tugrul, K.M. y Dursun, E. Some physical properties of sugarbeet seed. Journal of Stored 
Ancco, T. et al., Efecto de los tiempos de cosecha y almac. en solución hipertónica sobre el coef. de expansión volumétrica, vol., esfericidad y área superf. de los granos de maíz.

Products Research 2007; 43: 149-155

Floury J., Le Bail, Q.T. Pham. 2008. A three-dimensional numerical simulation of the osmotic dehydration of mango and effect of freezing on the mass transfer rates. Journal of Food Engineering 851-11.

Genina Soto, Próspero y Altamirano Morales, Silvia Beatriz. 2005. Deshidratación osmótica de prismas de camote, manzana y papa. INCI. vol.30, no.8, p.485487. ISSN 0378-1844.

Gupta R.K., Das S.K., "Physical properties of sunflower seeds". Journal of Agricultural Engineering. Research. Vol 66,pp. 1-8, 1997.

Ibarz Albert, Barbosa- Cánovas Gustavo V. 2005. Tecnología de Alimentos. Operaciones unitarias en la Ingeniería de Alimentos. Ediciones MundiPrensa. ISBN: 84-8476-163-0.

Jain, R.K. and S. Bal, 1997. Physical properties of pearl millet.J. Agric. Eng. Res., 66: 85-91.

Le Maguer, M.; SHI, J. and Fernandez, C. Mass transfer behavior of plant tissues during osmotic dehydration.En: Food Science and Technology International. Vol. 9, No. 3 (2003); p. 187-192.

LUH, B. and J. WOODROOF. Commercial vegetable processing. Wesport, Conn AVI Publishing. $1975.745 \mathrm{p}$.
Mohsenin, Nuri N. Physical properties of food and agricultural materials, A Teaching Manual New York; 1886: P. 147.

Pagano A.M., Crozza D.E., "Modelling of expansion during adsortion for two oleaginous". Tercer CongresoIberoamericano de Ingeniería de Alimentos", Valencia, España, 2001.

Pagano A.M., Crozza D.E., "Modelling of expansion during adsortion for two oleaginous". Tercer Congreso Iberoamericano de Ingeniería de Alimentos", Valencia, España, 2001.

Sablani, S. S., and Rahman, M. S. 2003. Effect of syrup concentration, temperature and sample geometry on equilibrium distribution coefficients during osmotic dehydration of mango. Food Research International, 36, 65-71.

Torreggiani Danila. Osmotic dehydration in fruit and vegetable processing. Istituto Valorizazione Tecnologica dei Prodotti Agricoli, Via Venezian 26, 20133 Milano, Italy. Food Research International 26 (1993) 59-68.

Torreggiani, D., Giangiacomo, R., Bertolo, G. and Abbo, E. 1986. Ricerche sulla disidratazione osmotica della frutta.II. Idoneita varietale delle albicocche. Zndustria Conserve, 61, 226-32. 